\title{
CubeSail Displaced Orbit Design for Near Earth Object Observation
}

\author{
Yang Yang ${ }^{1}$, Xiaokui Yue ${ }^{1}$, Yong Li $^{3}$, Andrew G. Dempster ${ }^{2}$, Chris. Rizos ${ }^{3}$ \\ ${ }^{1}$ School of Astronautics, Northwestern Polytechnical University, Xi'an, China \\ ${ }^{2}$ School of Electrical Engineering \& Telecommunications, University of New South Wales, Sydney, Australia \\ ${ }^{3}$ School of Civil \& Environmental Engineering, University of New South Wales, Sydney, Australia
}

\author{
Email address: \\ yiyinfeixiong@gmail.com (Yang Yang)
}

\section{To cite this article:}

Yang Yang, Xiaokui Yue, Yong Li, Andrew G. Dempster, Chris. Rizos. CubeSail Displaced Orbit Design for Near Earth Object Observation. American Journal of Aerospace Engineering. Vol. 2, No. 1, 2015, pp. 1-5. doi: 10.11648/j.ajae.20150201.11

\begin{abstract}
Microsatellites known as "CubeSats" have recently been developed to enable comparatively inexpensive and timely access to space for small payloads. As a new standard for small satellites, the CubeSat has shown great promise for space applications such as earth observation, planetary science and space physics mission. In this paper a "CubeSail" mission a CubeSat deployed with a solar sail -for near earth object (NEO) observation is introduced. It is important to observe a NEO which may intersect or pass close to earth space before instigating any procedure for hazard avoidance. Furthermore, close observation of NEO may also be important for exploiting the new resources and exploring new living environment in outer space. This paper describes the concept of a large numbers of CubeSails deployed in the vicinity of the NEO for observation purposes. The dynamic model of the NEO-centreddis placed orbit in space is analysed. The solar radiation pressure on the sail can be utilised as propulsion to compensate for third body gravitational perturbation. To maintain the relative motion/position between a CubeSail and the NEO, periodic initial conditions are searched, which also must satisfy some observation mission constraints. A simulation study is carried out using the near earth asteroid Apophis 99942, discovered in recent years.
\end{abstract}

Keywords: CubeSail, Multi-Object Global Optimisation, Relative Motion, Apophis 99942

\section{Introduction}

The original concept of a "CubeSat" was proposed by Bob Twiggs of Stanford University, California, USA (currently at Morehead State University, Kentucky, USA) and Jordi PuigSuari of California Polytechnic University at San Luis Obispo, California, USA in $1999^{[1]}$. The CubeSat's low cost, short development period and global user community, combined with its value as a teaching tool, has made this a new standard for small satellites. CubeSat, as the name implies, is a cube-shaped satellite with a $10 \mathrm{~cm} \times 10 \mathrm{~cm} \times 10$ $\mathrm{cm}$ volume having a mass no greater than $1 \mathrm{~kg}$. This standard size is termed 1 unit (U). CubeSats are often configured in $1.5 \mathrm{U}, 2 \mathrm{U}, 3 \mathrm{U}$ and $6 \mathrm{U}$ sizes, maintaining the $1 \mathrm{U}$ shape ${ }^{[2]}$. In recent years CubeSats have attracted great interest for space applications such as Earth observations, planetary science and space physics missions. QB50 is a proposed international space network of 50 CubeSats for multi-point, in-situ measurements in the lower thermosphere and for re-entry research $^{[3]}$. And the Surrey Space Centre is currently developing a nano-solar sail mission based on the $3 \mathrm{U}$ CubeSat standard ${ }^{[4]}$. One of the primary objectives of the "CubeSail" mission is to demonstrate deployment of a $25 \mathrm{~m}^{2}$ solar sail for one year deployment in a low Earth orbit (LEO). NanoSail-D, a recent NASA mission that demonstrated successful deployment of a solar sail in LEO, is similar to the CubeSail project ${ }^{[5]}$. Both make use of the $3 \mathrm{U}$ CubeSat platform, and both have four-quadrant square sails supported by metallic booms. What's more, ideas of launching CubeSats into deep space and "beyond low earth orbit" orbits have been proposed more recently. Pergola introduced an innovative space mission devoted to the survey of the small Earth companion asteroid by means of nano platforms ${ }^{[6]}$.

A near Earth object (NEO) is an asteroid or comet with an orbit that intersects or passes near that of the Earth. The growing interest in these objects has led to an increasing number of missions to study NEOs, such as the sample return mission Hayabusa of $\mathrm{JAXA}^{[7]}$, impact or mission Deep Impact of $\mathrm{NASA}^{[8]}$ and possible deflector demonstrator mission Don Quixote of ESA ${ }^{[9]}$. Also many research work has been focusing on redirecting or deflecting the orbit of 
NEOs to avoid their impact on our Earth ${ }^{[10-12]}$.

The concept of multiple CubeSats with very large solar sails orbiting in the vicinity of NEOs is presented in this paper. A NEO-centred displaced orbit ${ }^{[13,14]}$ chosen for the CubeSats in deep space is analysed. As periodicity of motion is a prerequisite to minimise the use of propulsion for the relative motion maintenance and control, the initial conditions are searched using a multi-object goal optimisation algorithm. The perturbation due to solar radiation pressure and third body gravity effects on a CubeSail relative to the NEO is compared. Simulations were conducted using the Apophis 99942 near Earth asteroid as an example NEO.

\section{Displaced Orbit for CubeSail}

The non-Keplerian orbit displaced near a planet was researched by McInnes and co-workers, and their results are summarised in the paper [13]. In our case, a NEO-centred displaced orbit is chosen for the CubeSail observation mission, as shown in Figure 1.Such a displaced orbit can be maintained by suitably orienting the thrust direction so as to balance the centrifugal component of spacecraft acceleration.

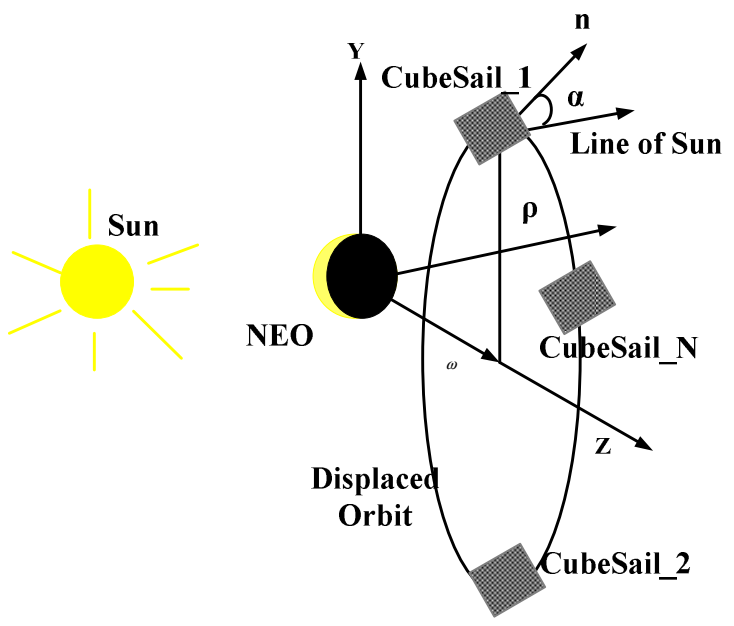

Fig. 1. NEO-centred displaced orbit ${ }^{[13]}$.

Firstly, the motion of the displaced orbit should be modelled for the CubeSail. In order to investigate the relative motion between the CubeSail and the NEO, the Hill frame is used: originating on the NEO, x-axis directed towards the radius vector of the NEO, z-axis pointing along the angular velocity of the NEO, and the $\mathrm{y}$-axis completing the right hand orthogonal triad (see Figure 2). In Figure 2, the $\boldsymbol{r}_{A} \boldsymbol{r}_{s c}$ are the absolute radius of the NEO and the spacecraft from the centre of the Sun respectively. $\rho$ is the relative radius vector.

In the Hill frame, the orbital mechanics for the CubeSail with respect to the NEO can be described (see Eq. (1)). The multi-body gravity effects and the solar radiation pressure perturbations are neglected in the initial study for simplicity, assuming that the CubeSail's motion is only influenced by the Sun. In the following section, however, the idea of utilising the negative solar radiation pressure perturbation as positive thrust to compensate for the third body gravity effect of the NEO will be discussed.

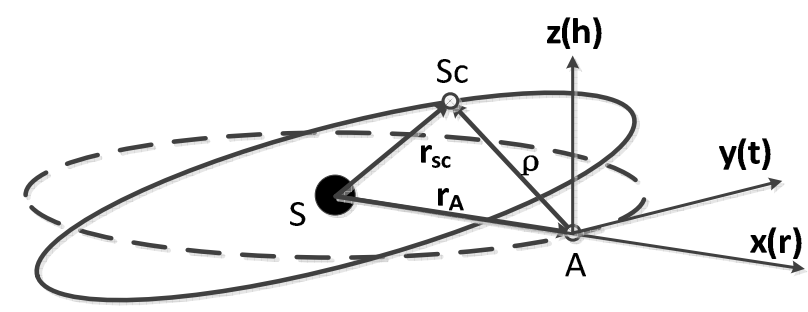

Fig. 2. The Hill coordinate system for relative motion.

$$
\begin{aligned}
& \ddot{x}=2 \dot{f}_{A}\left(\dot{y}-y \frac{\dot{r}_{A}}{r_{A}}\right)+x \dot{f}_{A}^{2}+\frac{\mu_{S}}{r_{A}^{2}}-\frac{\mu_{S}}{r_{s c}^{3}}\left(r_{A}+x\right)+u_{x} \\
& \ddot{y}=-2 \dot{f}_{A}\left(\dot{x}-x \frac{\dot{r}_{A}}{r_{A}}\right)+y \dot{f}_{A}^{2}-\frac{\mu_{S}}{r_{s c}^{3}} y+u_{y} \\
& \ddot{z}=-\frac{\mu_{S}}{r_{s c}^{3}} z+u_{z}
\end{aligned}
$$

Where $f_{A}$ is the true anomaly; $r_{A}$ is the radius of the NEO in the sun-centred coordinate system; $\mu_{S}$ is the gravity coefficient of the sun; $r_{s c}$ is the distance between NEO and the CubeSail; $\left[\mu_{\mathrm{x}} ; \mu_{y} ; \mu_{z}\right]$ is the thrust acting on the CubeSail.

\section{Initial Conditions for Periodic Relative Motion}

Since the CubeSats are of small size and relatively simple structure, there is limited capacity for complex payloads. Hence multiple CubeSats are proposed, working together to fulfil the observation mission. Several considerations should be taken into account in the mission design. Firstly, the distance between the CubeSail and the NEO should be as short as possible for better imaging or sensing. Secondly, the radius of the displaced orbit should be large enough in order to avoid possible collisions between multiple CubeSails. Finally, the CubeSails should probably fly out of the Hill sphere of the NEO to escape the gravity effect of the NEO. The limitation conditions are formulated as shown in Eq. (2) - Eq. (4):

$$
\begin{gathered}
\min J_{D 1}=\rho_{T} \\
\min J_{D 2}=-\sqrt{{x_{T}{ }^{2}+z_{T}{ }^{2}}^{2 / 5}} \\
C_{\text {ineq }}=\rho_{T}-r_{\text {lim }}>0, \quad r_{\text {lim }}=a_{A}\left(1-e_{A}\right)\left(\frac{m_{A}}{m_{S}}\right)^{2 / 5}
\end{gathered}
$$

Where $r_{\text {lim }}$ is the radius of the NEO's Hill sphere, inside of which the NEO dominates the attraction of the satellites; $a_{A}$, $e_{A}$ are the semi-major axis and the eccentricity of the NEO 
orbit; $m_{A}, m_{S}$ are the mass of the NEO and the Sun, respectively. For the Apophis 99942 near Earth asteroid, $r_{\lim } \approx 1.5592 \times 10^{3} \mathrm{~m}$. Three equations mentioned above are necessary to be considered in the initial value search for the dynamic equations (Eq. (1)) of the CubeSail.

Above all, the initial conditions should satisfy the periodicity so as to ensure that fuel for the formation maintenance is conserved, hence extending the life of the CubeSat. The formula for the periodicity limitation is:

$$
\min J_{D 0}=\sqrt{\left(x_{T}-x_{0}\right)^{2}+\left(y_{T}-y_{0}\right)^{2}+\left(z_{T}-z_{0}\right)^{2}}
$$

In this paper, the Matlab ${ }^{\circledR}$ Optimization Tool is used to execute the above multi-objective optimisation, in which the solver adopts the Genetic Algorithm. A family of optimal results can be obtained as the Pareto results. One set of initial values is chosen according to Eq. (5) for one CubeSail in the displaced orbit:

$$
X^{\text {opt }}=\left[\begin{array}{c}
x_{0}=-365.95 \mathrm{~m} \\
y_{0}=663.20 \mathrm{~m} \\
z_{0}=1511.52 \mathrm{~m} \\
v_{x 0}=1.9257 \times 10^{-4} \mathrm{~m} / \mathrm{s} \\
v_{y 0}=1.7214 \times 10^{-4} \mathrm{~m} / \mathrm{s} \\
v_{z 0}=-1.3970 \times 10^{-4} \mathrm{~m} / \mathrm{s}
\end{array}\right]
$$

\section{Solar Radiation Pressure for Thrust Compensation}

When considering the third body gravity effect from the NEO, extra propulsion should be added to maintain the CubeSat orbit. The solar radiation pressure on the solar sail of the CubeSat can provide some valid thrust. Assume a spherical and homogenous gravity field for the asteroid, a more precise relative dynamics equation is:

$$
\mathrm{u}_{\text {pert }}=\mu_{A}\left(\frac{\rho}{\rho^{3}}-\frac{T_{I}^{H} \mathrm{r}_{A}^{I}}{r_{A}{ }^{3}}\right)+\frac{\mathrm{F}_{S R P}}{m_{A}}
$$

Here $\rho=\sqrt{x^{2}+y^{2}+z^{2}}$ is the relative distance between the CubeSail and the NEO; $\mu_{A}$ is the gravity constant of the NEO; and $\boldsymbol{F}_{s}=\left[\begin{array}{lll}F_{S R P x} & F_{S R P y} & F_{S R P z}\end{array}\right]^{T}$ is the force vector due to the solar radiation pressure on the sail. The transformation matrix between the initial and Hill frames is:

$$
T_{I}^{H}=\mathrm{R}_{3}\left(\omega_{A}+f_{A}\right) \mathrm{R}_{1}\left(I_{A}\right) \mathrm{R}_{3}\left(\Omega_{A}\right)
$$

where the $\mathrm{R}_{1}, \mathrm{R}_{3}$ denote the rotation matrix along the $\mathrm{x}$-axis and z-axis, respectively. Some artificial equilibrium points exist where both the acceleration and the velocity are zero, namely $\left[\begin{array}{ccc}\dot{x} & \dot{y} & \dot{z}\end{array}\right]^{T}=0_{3 \times 1},\left[\begin{array}{lll}\ddot{x} & \ddot{y} & \ddot{z}\end{array}\right]^{T}=0_{3 \times 1}$, and obtained by a search in the solution domain ${ }^{[15]}$. An important parameter of the CubeSail design is the area of the solar sail. Currently a $5 m \times 5 m$ CubeSail has been developed by the Surrey Space Centre and has completed testing for ground deployment ${ }^{[3]}$. For the simulations described in this paper, this value for the sail area is used. The force due to the solar radiation pressure is given by the following model. In order to observe the NEO, the CubeSail is assumed to point towards the NEO, which leads to an angle $\alpha$ between the Sun light vector los and the solar radiation force direction $\mathrm{n}$ (see Figure 3). The force is expressed in Eq. (9).
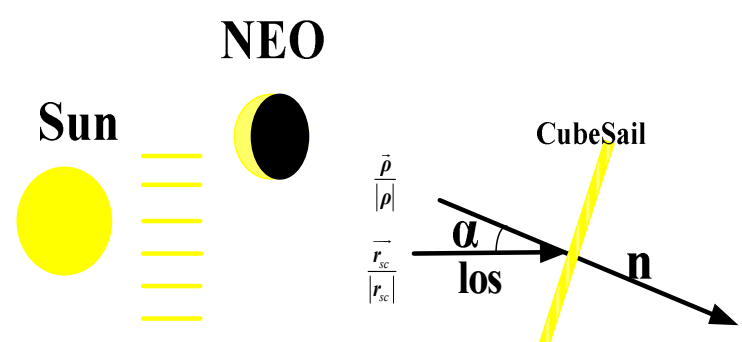

Fig. 3. The sun light vector on the solar sail.

$$
\begin{aligned}
& \mathrm{F}_{S R P}=\sigma_{S} P_{S R} A \cos ^{2} \alpha \mathrm{n} \\
& P_{S R}=\frac{P_{0}}{c}\left(\frac{A_{u}}{\rho}\right)^{2} \\
& \alpha=\pi-\arcsin \frac{\rho \cdot \mathrm{r}_{s c}}{|\rho| \cdot\left|\mathrm{r}_{s c}\right|} \\
& \mathrm{n}=\frac{\rho}{|\rho|}
\end{aligned}
$$

where $\sigma_{S}$ is the efficiency of the solar sail; $P_{S R}$ is the radiation pressure at the CubeSail surface $\left(N / \mathrm{m}^{2}\right) ; A$ is the Sun-facing cross-sectional area $\left(\mathrm{m}^{2}\right) ; A_{u}$ is the astronomical unit $\left(1.4959787 \times 10^{11} \mathrm{~m}\right) ; P_{0}$ is the intensity of solar radiation $\left(w / m^{2}\right) ; c$ is the speed of light $(m / s)$.

\section{Simulation and Results}

Apophis 99942 is a near Earth asteroid discovered in 2004, which will pass close to the Earth in 2029, may pose subsequent impact threats in 2036, 2037 or later ${ }^{[16]}$. This paper will discuss the challenge of Apophis observation using CubeSail concept. The orbital parameters of the Apophis are listed in the Table 1.

Table 1. Estimated and observed orbital parameters of Apophis $99942^{[17]}$

\begin{tabular}{lll}
\hline Orbital Elements & & Measurements \\
\hline Semi-major axis & $a$ & $0.922300 \mathrm{AU}$ \\
Eccentricity & $e$ & 0.191076 \\
Inclination & $i$ & $3.33196^{\circ}$ \\
Right Argument of Ascending & $\Omega$ & $204.430410^{\circ}$ \\
\hdashline Argument of periapsis & $\omega$ & $126.424477^{\circ}$ \\
Period & $T$ & $323.596917 \mathrm{~d}$ \\
Mean motion & $n$ & $1.112745^{\circ} / \mathrm{d}$ \\
\hline
\end{tabular}


According to the initial conditions in Eq. (6), the CubeSail orbit is propagated to verify the periodicity of the relative motion. The initial epoch for the Appophis is 13:37:00 on 13 April, 2031, which is 5 years ahead of the potential impact. The simulation time lasts for 5 orbital periods of the Apophis NEO. The relative motion is illustrated in Figure 4. It is periodic, closed and bounded.

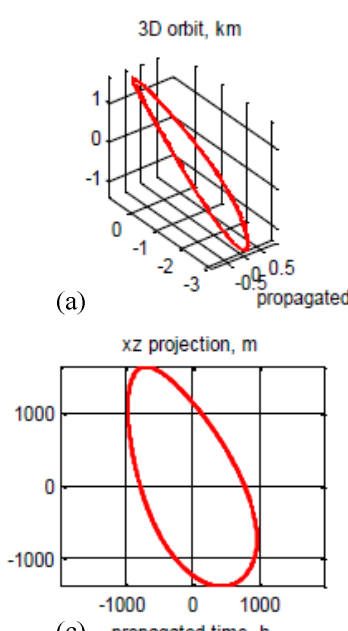

(c) propagated time, $\mathrm{h}$

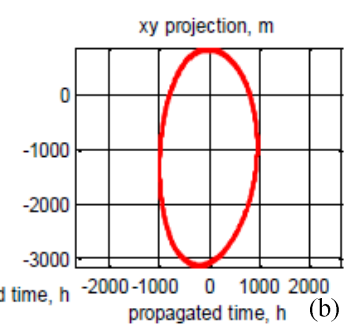

yz projection, $m$

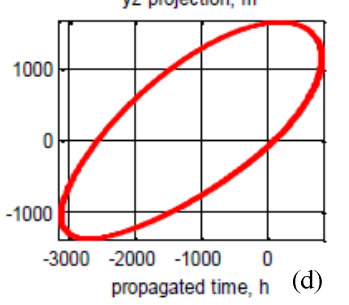

Fig. 4. Relative orbit between the CubeSail and the NEO.
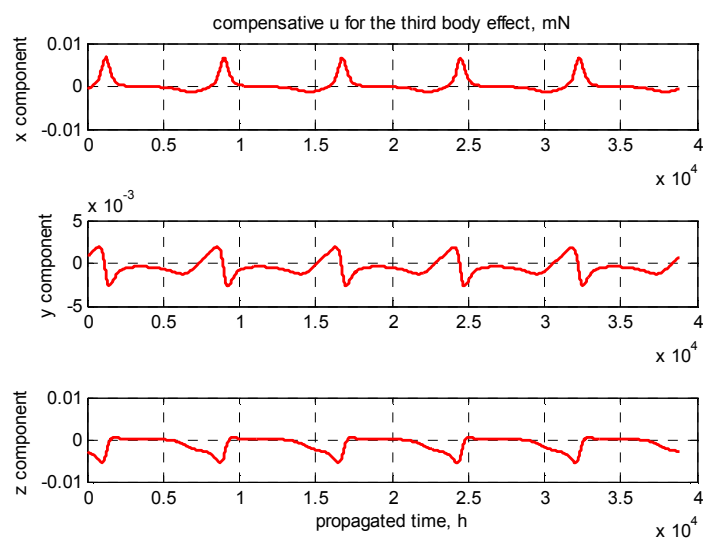

Fig. 5. Third body gravity perturbations due to the NEO.

As discussed above, third body gravity perturbations on the CubeSails cannot be ignored, as they will damage the periodicity of the relative motion and cause the orbit to drift. Figure 5 shows the extra thrust needed for the third body gravity perturbations from the NEO for the three axes with the order of $1 \mathrm{mN}$. For the CubeSails, the solar radiation pressure can be used as positive continuous thrust to compensate for the third body gravity perturbations. Using Eq. (9), the accelerations induced by the solar radiation pressure can be calculated. The components of solar radiation pressure acceleration are shown in Figure 6, from which one can see that the solar radiation pressure is one order of magnitude greater than the third body gravity perturbations, indicating that it is possible to compensate for these perturbations if the spacecraft attitude (relative to the Sun) are appropriately selected and controlled. The total disturbing forces in the three directions are shown in Figure 6.
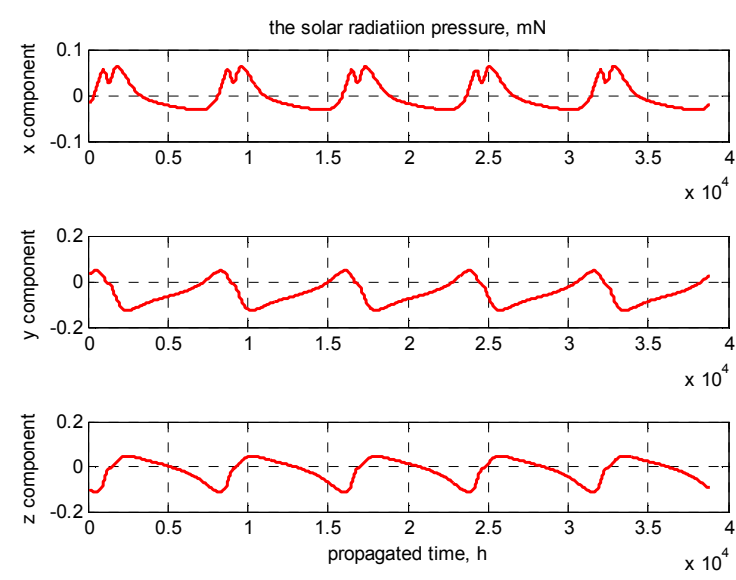

Fig. 6. The solar radiation pressure perturbation.
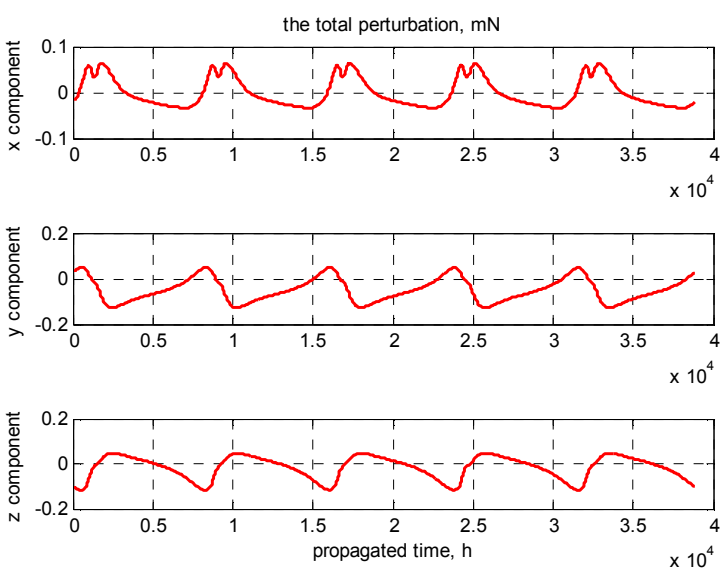

Fig. 7. The total perturbation.

\section{Concluding Remarks}

This paper presented a concept of NEO observation using a proposed Cube Sail spacecraft flying near the NEO. The NEO-centred displaced orbit mechanics was analysed. The initial conditions were searched using a multi-object goal genetic optimisation algorithm. A precise dynamic model for the Cube Sail relative to the NEO was defined, and the solar radiation pressure and the third body gravity perturbations were discussed. Utilising the solar radiation pressure as a form of propulsion for CubeSats shows great potential.

\section{Acknowledgement}

China Scholarship Council (CSC) is gratefully acknowledged for supporting the author's visiting period at the School of Surveying and Geospatial Engineering, the University of New South Wales. Also the author appreciates the help from the researchers in the Australian Centre for Space Engineering Research. 


\section{References}

[1] Pang, A.S.-K. and B. Twiggs, Citizen Satellites. Scientific American, 2011. 304(2): p. 48-53.

[2] Shiroma, W., et al., CubeSats: A bright future for nanosatellites. Central European Journal of Engineering, 2011. 1(1): p. 9-15.

[3] Gill, E., et al. Formation Flying to Enhance the QB50 Space Network. 2010.

[4] Lappas, V., et al., CubeSail: A low cost CubeSat based solar sail demonstration mission. Advances in Space Research, 2011. 48(11): p. 1890-1901.

[5] Johnson, L., et al., Status of solar sail technology within NASA. Advances in Space Research, 2011. 48(11): p. 16871694.

[6] Pergola, P., Small satellite survey mission to the second Earth moon. Advances in Space Research, 2013. 52(9): p. 16221633.

[7] Kawaguchi, J.i., A. Fujiwara, and T. Uesugi, Hayabusa-Its technology and science accomplishment summary and Hayabusa-2. Acta Astronautica, 2008. 62(10-11): p. 639-647.

[8] A'Hearn, M., et al., Deep impact: excavating comet Tempel 1. Science, 2005. 310(5746): p. 258-264.

[9] Gálvez, A. and I. Carnelli, ESA’S DON QUIJOTE MISSION: AN OPPORTUNITY FOR THE INVESTIGATION OF AN ARTIFICIAL IMPACT CRATER ON AN ASTEROID. 2006.

[10] Eneev, T.M., R.Z. Akhmetshin, and G.B. Efimov, On the asteroid hazard. Cosmic Research, 2012. 50(2): p. 93-102.

[11] Gates, M. and L. Johnson, NASA's Asteroid Redirect Mission, in Handbook of Cosmic Hazards and Planetary Defense, F. Allahdadi and J.N. Pelton, Editors. 2014, Springer International Publishing. p. 1-7.

[12] Sugimoto, Y., et al., Hazardous near Earth asteroid mitigation campaign planning based on uncertain information on fundamental asteroid characteristics. Acta Astronautica, 2014. 103(0): p. 333-357.

[13] McInnes, C.R., Solar sail mission applications for nonKeplerian orbits. Acta Astronautica, 1999. 45(4-9): p. 567-575.

[14] McInnes, C.R., Passive Control of Displaced Solar Sail Orbit. Journal of Guidance, Control, and Dynamics, 1998. 21(6).

[15] Vasile, M., A multi-mirror solution for the deflection of dangerous NEOS. Communications in Nonlinear Science and Numerical Simulation, 2009. 14(12): p. 4139-4152.

[16] Ferraz-Mello, S. and J. Fernández, Potential impact detection for near-Earth asteroids: The case of 99942 Apophis (2004 MN4). 2005.

[17] Vasile, M., C.A. Maddock, and G. Radice. Mirror formation control in the vicinity of an asteroid. in AIAA/AAS Astrodynamics Specialist Conference and Exhibit. 2008. Honolulu, Hawaii. 